\title{
MINIMAL GRAPHS WITH DISJOINT DOMINATING AND PAIRED-DOMINATING SETS
}

\author{
Michael A. Henning \\ Department of Pure and Applied Mathematics \\ University of Johannesburg \\ Auckland Park 2006, South Africa \\ e-mail: mahenning@uj.ac.za \\ AND \\ JERZY TOPP \\ The State University of Applied Sciences in Elblag, Poland \\ e-mail: j.topp@inf.ug.edu.pl
}

\begin{abstract}
A subset $D \subseteq V_{G}$ is a dominating set of $G$ if every vertex in $V_{G}-D$ has a neighbor in $D$, while $D$ is a paired-dominating set of $G$ if $D$ is a dominating set and the subgraph induced by $D$ contains a perfect matching. A graph $G$ is a $D P D P$-graph if it has a pair $(D, P)$ of disjoint sets of vertices of $G$ such that $D$ is a dominating set and $P$ is a paired-dominating set of $G$. The study of the $D P D P$-graphs was initiated by Southey and Henning [Cent. Eur. J. Math. 8 (2010) 459-467; J. Comb. Optim. 22 (2011) 217-234]. In this paper, we provide conditions which ensure that a graph is a $D P D P$-graph. In particular, we characterize the minimal $D P D P$-graphs.
\end{abstract}

Keywords: domination, paired-domination.

2010 Mathematics Subject Classification: 05C69, 05C85.

\section{REFERENCES}

[1] V. Anusuya and R. Kala, A note on disjoint dominating sets in graphs, Int. J. Contemp. Math. Sci. 7 (2012) 2099-2110.

[2] I. Broere, M. Dorfling, W. Goddard, J.H. Hattingh, M.A. Henning and E. Ungerer, Augmenting trees to have two disjoint total dominating sets, Bull. Inst. Combin. Appl. 42 (2004) 12-18. 
[3] G. Chartrand, L. Lesniak and P. Zhang, Graphs and Digraphs (CRC Press, Boca Raton, 2016)

[4] P. Delgado, W.J. Desormeaux and T.W. Haynes, Partitioning the vertices of a graph into two total dominating sets, Quaest. Math. 39 (2016) 863-873. doi:10.2989/16073606.2016.1188862

[5] W.J. Desormeaux, T.W. Haynes and M.A. Henning, Partitioning the vertices of a cubic graph into two total dominating sets, Discrete Appl. Math. 223 (2017) $52-63$. doi:10.1016/j.dam.2017.01.032

[6] M. Dorfling, W. Goddard, J.H. Hattingh and M.A. Henning, Augmenting a graph of minimum degree 2 to have two disjoint total dominating sets, Discrete Math. 300 (2005) 82-90.

doi:10.1016/j.disc.2005.06.020

[7] T.W. Haynes and M.A. Henning, Trees with two disjoint minimum independent dominating sets, Discrete Math. 304 (2005) 69-78. doi:10.1016/j.disc.2005.09.012

[8] S.M. Hedetniemi, S.T. Hedetniemi, R.C. Laskar, L. Markus and P.J. Slater, Disjoint dominating sets in graphs, in: Proc. ICDM 2006, Ramanujan Math. Soc. Lect. Notes Ser. 7 (2008) 87-100.

[9] P. Heggernes and J.A. Telle, Partitioning graphs into generalized dominating sets, Nordic J. Comput. 5 (1988) 128-142.

[10] M.A. Henning, C. Löwenstein and D. Rautenbach, Remarks about disjoint dominating sets, Discrete Math. 309 (2009) 6451-6458. doi:10.1016/j.disc.2009.06.017

[11] M.A. Henning, C. Löwenstein and D. Rautenbach, Partitioning a graph into a dominating set, a total dominating set, and something else, Discuss. Math. Graph Theory 30 (2010) 563-574. doi:10.7151/dmgt.1514

[12] M.A. Henning, C. Löwenstein and D. Rautenbach, An independent dominating set in the complement of a minimum dominating set of a tree, Appl. Math. Lett. 23 (2010) 79-81. doi:10.1016/j.aml.2009.08.008

[13] M.A. Henning, C. Löwenstein, D. Rautenbach and J. Southey, Disjoint dominating and total dominating sets in graphs, Discrete Appl. Math. 158 (2010) 1615-1623. doi:10.1016/j.dam.2010.06.004

[14] M.A. Henning and A.J. Marcon, Semitotal domination in graphs: Partition and algorithmic results, Util. Math. 106 (2018) 165-184.

[15] M.A. Henning and D.F. Rall, On graphs with disjoint dominating and 2-dominating sets, Discuss. Math. Graph Theory 33 (2013) 139-146.

doi:10.7151/dmgt.1652 
[16] M.A. Henning and J. Southey, A note on graphs with disjoint dominating and total dominating sets, Ars Combin. 89 (2008) 159-162.

[17] M.A. Henning and J. Southey, A characterization of graphs with disjoint dominating and total dominating sets, Quaest. Math. 32 (2009) 119-129.

doi:10.2989/QM.2009.32.1.10.712

[18] M.A. Henning and A. Yeo, Total Domination in Graphs (Springer Monographs in Mathematics, Springer, 2013). doi:10.1007/978-1-4614-6525-6

[19] E.M. Kiunisala and F.P. Jamil, On pairs of disjoint dominating sets in a graph, Int. J. Math. Anal. 10 (2016) 623-637. doi:10.12988/ijma.2016.6343

[20] V.R. Kulli and S.C. Sigarkanti, Inverse domination in graphs, Nat. Acad. Sci. Lett. 14 (1991) 473-475.

[21] C. Löwenstein and D. Rautenbach, Pairs of disjoint dominating sets and the minimum degree of graphs, Graphs Combin. 26 (2010) 407-424. doi:10.1007/s00373-010-0918-9

[22] M. Miotk, J. Topp and P. Żyliński, Disjoint dominating and 2-dominating sets in graphs, Discrete Optim. 35 (2020) 100553. doi:10.1016/j.disopt.2019.100553

[23] O. Ore, Theory of Graphs (Providence, RI, 1962).

[24] J. Southey and M.A. Henning, Graphs with disjoint dominating and paireddominating sets, Cent. Eur. J. Math. 8 (2010) 459-467. doi:10.2478/s11533-010-0033-4

[25] J. Southey and M.A. Henning, Dominating and total dominating partitions in cubic graphs, Cent. Eur. J. Math. 9 (2011) 699-708. doi:10.2478/s11533-011-0014-2

[26] J. Southey and M.A. Henning, A characterization of graphs with disjoint dominating and paired-dominating sets, J. Comb. Optim. 22 (2011) 217-234. doi:10.1007/s10878-009-9274-1

Received 20 November 2019

Revised 27 April 2020

Accepted 27 April 2020 